\title{
Antimycobacterial Effect and Mechanisms of Monoterpenoid, Perillyl Alcohol
}

\author{
Venkata Saibabu", Rahul Pal ${ }^{\#}$, Saif Hameed* and Zeeshan Fatima* \\ Amity Institute of Biotechnology, Amity University Haryana, Gurgaon (Manesar)-122413, \\ ${ }^{*}$ Authors are equally contributed
}

Received: November 8, 2016; Accepted: December 1, 2016; Published: December 5, 2016

*Corresponding author: Zeeshan Fatima and Saif Hameed, Amity Institute of Biotechnology, Amity University, Haryana, Gurgaon (Manesar)-122413, India, Phone: +91-124-2337015, Ext: 1205; E-mail: drzeeshanfatima@gmail.com, saifhameed@yahoo.co.in

\begin{abstract}
Continuous deployment of antitubercular drugs has led to emergence of Multi-Drug Resistance (MDR) acquired by Mycobacterium Tuberculosis (MTB) and warrants immediate search for novel drugs and mechanisms. Here, we investigated the anti-mycobacterial effect of a natural monoterpene, Perillyl Alchol (PA) against Mycobacterium smegmatis, a surrogate for MTB. We observed that antimycobacterial activity of PA as observed from broth microdilution and spot assays was $350 \mathrm{\mu gml}^{-1}$. Insight studies into possible mechanisms suggested that PA targets cell membrane of mycobacteria independent of drug efflux transporter activity. Membrane damaging effect of PA was revealed by enhanced passive diffusion, permeability across the cell membrane and hypersensitivity against membrane perturbing agent, SDS. Additionally, PA also affects cell surface phenotypes as displayed by altered colony morphology impaired sliding motility and enhanced cell sedimentation rate. We further explored that PA inhibited biofilm formation, cell aggregation in liquid cultures and adherence of mycobacterium to the buccal epithelial cells which are crucial virulence traits. In addition, PA leads to hypersensitive response in presence of $\mathrm{EtBr}$, a DNA damaging agent, suggesting possible defect in DNA repair machinery. Together, this study for the first time establishes the anti-mycobacterial potential of PA that may be further exploited for improving the therapeutic strategies.
\end{abstract}

Keywords: Tuberculosis; Terpene; Membrane; Biofilm; Cell adherence

\section{Introduction}

Tuberculosis (TB) is a chronic communicable disease causing high morbidity and mortality in under developed and developing countries [1]. Despite, one third of the world's population being affected by Mycobacterium tuberculosis (MTB), only 5\% of infected individual develops symptoms of TB [2]. Several quandaries are associated with increased use of standard drugs including side effects and high cost [3]. In addition, emergence of multi drug resistance (MDR-TB), extensive drug resistance (XDR-TB) and advent of HIV-TB have compounded the problem demanding new and effective drugs to treat TB [4,5]. Plants represent a rich reservoir of biologically active compounds including polyphenols, terpenoids and alkaloids [6]. In recent years, large numbers of extracts and pure compounds have been studied against MTB and other Mycobacterium species including MDR strains which have shown promising potential [7].

Perillyl Alcohol (PA) or p-Mentha-1, 8-diene-7-ol (Figure 1) is a monocyclic monoterpene synthesized by the mevalonate pathway in plants which is stable at room temperature. It is found in the essential oil of various plant species such as cherries, mints, lemon and ginger grass [8]. Recent findings have shown that PA has anticancer, antioxidant and anti-inflammatory properties and used in the treatment of neurological complications and lung disorders [9]. PA is approved by the U.S. Food and Drug Administration as a food additive that can be safely consumed by human displaying its non-toxic nature [10]. Our group has already deciphered the antifungal potential of PA against one of the most prevalent human fungal pathogen, Candida albicans [10]. Currently, there is no published report demonstrating the anti-mycobacterial activity of PA hence the present study aimed to decipher the anti-mycobacterial potential of PA against mycobacterial surrogate model for MTB, Mycobacterium smegmatis. We explored that anti-mycobacterial action of PA is possibly linked to disruption of membrane homeostasis, inhibited biofilm formation and cell adherence and defect in DNA repair response machinery.

\section{Materials and Methods}

All Media chemicals Middlebrook 7H9 broth, Middlebrook 7H10 agar, Albumin/Dextrose/Catalase (ADC), Oleic Acid/ Albumin/Dextrose/Catalase (OADC) supplements was purchased from BD Biosciences (USA). Perillyl alcohol (purity 96\%), Isoniazid (INH), Tween-80, Nitrocefin were purchased from Sigma-Aldrich (St. Louis, MO, USA). Ethidium Bromide (EtBr), Dinitrophenol (2, 4, DNP), and crystal violet were purchased from Himedia (Mumbai, India). Dimethyl sulfoxide<smiles>C=C(C)[C@H]1CC=C(CO)CC1</smiles>

Figure 1: Chemical structure of PA 
(DMSO), Potassium chloride (KCl), Sodium chloride ( $\mathrm{NaCl}$ ), diSodium Hydrogen Orthophosphate $\left(\mathrm{Na}_{2} \mathrm{HPO}_{4}\right)$, Potassium Dihydrogen Orthophosphate $\left(\mathrm{KH}_{2} \mathrm{PO}_{4}\right)$, Sodium Dodecyl Sulphate (SDS), Glycerol, D-Glucose were obtained from Fischer Scientific. Agarose was purchased from $\mathrm{CDH}$, India.

\section{Growth conditions}

M. smegmatis $\mathrm{mc}^{2} 155$ and M. tuberculosis (MTB) $\mathrm{H}_{37} \mathrm{Rv}$ were grown in Middlebrook 7H9 (BD Biosciences) broth supplemented with $0.05 \%$ tween-80 (SIGMA), 10\% Albumin/Dextrose/Catalase (ADC; BD Difco), and $0.2 \%$ glycerol (Fischer Scientific) in 100 $\mathrm{mL}$ culture flasks (Schott Duran) and incubated at $37^{\circ} \mathrm{C}$ and on Middlebrook 7H10 (BD Biosciences) agar media supplemented with $10 \%$ (v/ v) Oleic Acid/ Albumin/Dextrose/Catalase (OADC; BD Difco) for solid agar to grow at $37^{\circ} \mathrm{C}$. Stock cultures of logphase cells were maintained in $30 \%$ glycerol and stored at $-80^{\circ} \mathrm{C}$.

\section{Drug susceptibility testing}

Drug susceptibility was tested using Minimal Inhibitory Concentration (MIC) and spot assay as described below:

Minimum Inhibitory Concentration (MIC): MIC was determined by broth dilution method as described in method M27-A3 from the Clinical and Laboratory Standards Institute [11]. Briefly, $100 \mu \mathrm{l}$ of Middlebrook 7H9 broth supplemented with OADC enrichment $0.5 \%$ (v/ v) glycerol $0.05 \%$ (v/ v) Tween 80 was placed at each well of the 96 wells plate following with the addition of the drug with the remaining media and then subsequently it was serially diluted 1:2.100 $\mu \mathrm{l}$ of cell suspension in normal saline to an Optical density $\left(\mathrm{OD}_{600}\right) 0.1$ was added to each well of the plate. Plates were incubated at $37^{\circ} \mathrm{C}$ for 48 hours. For the MTB cultures, plates were incubated at $37^{\circ} \mathrm{C}$ for 7 days. The $\mathrm{MIC}_{80}$ values were evaluated by observing the $\mathrm{OD}_{600}$ in a microplate reader The $\mathrm{MIC}_{80}$ was defined as the concentration at which the $80 \%$ of the growth was inhibited compared with the controls.

Spot assay: Spot assays for the strains were determined using a method as described previously $[12,13]$. Briefly, for the spot assay $5 \mu \mathrm{L}$ of fivefold serial dilutions of each $M$. smegmatis culture (each with cells suspended in normal saline to an $\mathrm{OD}_{600} \mathrm{~nm}$ of 0.1) was spotted onto Middlebrook 7H10 agars supplemented with OADC enrichment $0.5 \%$ (v/ v) glycerol $0.05 \%(\mathrm{v} / \mathrm{v})$ plates. Growth difference was measured after incubation at $37^{\circ} \mathrm{C}$ for 48 hours.

\section{EtBr efflux and passive diffusion}

The efflux of EtBr was determined by using protocol described previously [13]. Briefly, approximately $1 \times 10^{6}$ cells were incubated until exponential phage in the absence of drug (control) and in presence of PA at its sub-inhibitory concentration $\left(175 \mu \mathrm{gmL}^{-1}\right)$ determined by the growth curve experiments (data not shown). Cells were pelleted, washed twice with PhosphateBuffered Saline (PBS) (without glucose), and resuspended as a 2\% cell suspension. The cells were then de-energized with an efflux pump inhibitor 2, 4 DNP (20 $\left.\mathrm{ggml}^{-1}\right)$ in PBS (without glucose). The de-energized cells were pelleted, washed, and then resuspended as a $2 \%$ cell suspension $(\mathrm{w} / \mathrm{v})$ in PBS without glucose, to which $\mathrm{EtBr}$ was added at a final concentration of $4 \mathrm{\mu gml}^{-1}$ and incubated for $45 \mathrm{~min}$ at $30^{\circ} \mathrm{C}$. The equilibrated cells with $\mathrm{EtBr}$ were then washed and resuspended as a $2 \%$ cell suspension (w/v) in PBS (with glucose $0.4 \%$ ) for EtBr efflux and PBS (w/o glucose) for passive diffusion respectively. Samples with a volume of $2 \mathrm{ml}$ were withdrawn at the indicated time points as mentioned in the figure legend and centrifuged at 10,000 rpm for $1 \mathrm{~min}$. The supernatant was collected, and absorption was measured at 285 $\mathrm{nm}$. Glucose-free controls were included in all the experiments.

\section{Permeability assay}

The cell membrane permeability of $M$. smegmatis was determined by measuring the hydrolysis of the chromogenic cephalosporin nitrocefin by whole cells as described previously [13]. Briefly, cells were grown overnight at $37^{\circ} \mathrm{C}$ in the absence (control) and presence of PA at its sub-inhibitory concentration with continuous shaking. Cells were then equalized with cold 1x Phosphate-Buffered Saline (PBS) buffer (pH 7.4). Nitrocefin was added to a final concentration of $50 \mu$ to the aliquot $2 \mathrm{~mL}$ in $1 \mathrm{x}$ PBS (pH 7.4) and hydrolysis was monitored as a change in absorbance at $486 \mathrm{~nm}$ at indicated time points.

\section{Colony Morphology and Sliding Motility}

Colony morphology and sliding motility were determined as described elsewhere [14]. Briefly, cells were plated on MB7H10 agar plates supplemented with 10\% OADC (BD Difco) and incubated at $37^{\circ} \mathrm{C}$ for 2 to 4 days. Post incubation, images of the individual colonies were taken at 10x magnification. To score for sliding motility, cells were grown until stationary phase in MB7H9 medium supplemented with 10\% OADC (BD Difco) in the absence (control) and presence of PA at its sub-inhibitory concentration. $3 \mu \mathrm{l}$ of the culture was spotted in the middle of MB7H10 plates solidified with $0.3 \%$ agarose without any carbon source. The plates were incubated at $37^{\circ} \mathrm{C}$ for 4 days.

\section{Cell sedimentation assay}

Cell sedimentation assay was performed as described elsewhere [14]. Cultures at $\mathrm{OD}_{600} \sim 1.0-1.4$ of the control and cells treated with sub-inhibitory concentration $(175 \mu \mathrm{g} / \mathrm{ml})$ of PA in middle brook media supplemented with OADC were adjusted in triplicate to $\mathrm{OD}_{590} \sim 1.0$ and kept unshaken at $37^{\circ} \mathrm{C}$. At 3 and 22 hours, the upper $1 \mathrm{ml}$ was removed for $\mathrm{OD}_{590}$ measurements.

\section{Biofilm formation}

Biofilm forming potentially analyzed qualitatively and quantitatively using microtiter-plate method as described previously [14,15]. Briefly, M. smegmatis culture was grown overnight at $37^{\circ} \mathrm{C}$ in middle brook media. $100 \mu \mathrm{l}$ of media was placed at each well of the 96 well plates in absence and presence of PA. $0.1 \mathrm{OD}_{600}$ cultures were 1:100 diluted in the middle brook media and $100 \mu \mathrm{l}$ of each diluted culture was pipetted in each well of 96 -well flat bottom microtiter plate and incubated at $37^{\circ} \mathrm{C}$ for 48 hours. The wells were rinsed with water and $125 \mu \mathrm{l}$ of $0.1 \%$ solution of the Crystal Violet (CV) was added. Plates were incubated at $10 \mathrm{~min}$ followed by washing twice with distilled 
water and observed under the light microscope at 100x. For quantitative assay of biofilm, $200 \mu \mathrm{l}$ of $95 \%$ ethanol was added to each crystal violet stained well and plates were incubated for $10 \mathrm{~min}$ at room temperature. Contents of each well were mixed by pipette, and then $125 \mu \mathrm{l}$ of the Crystal Violet/Ethanol solution was transferred from each well to a separate well of an optically clear flat bottom 96-well plate and OD was measured at $600 \mathrm{~nm}$ using spectrophotometer. Inhibition of biofilm was calculated as percentage inhibition/reduction in growth.

\section{Aggregation assay}

Aggregation assay was performed as described elsewhere [14]. Briefly, cells were grown at $37^{\circ} \mathrm{C}$ for $24 \mathrm{~h}$ in MB7H9 broth supplemented with 10\% OADC (BD Difco) with or without PA. The cells were harvested by centrifugation and suspended in PBS to 0.5 Optical Density (O.D.) units at $600 \mathrm{~nm} .2 \mathrm{ml}$ bacterial suspension were placed in each tube and centrifuged. The cells were then resuspended in their culture supernatant fluids. After incubation at $37^{\circ} \mathrm{C}$ for $2 \mathrm{~h}, 1 \mathrm{ml}$ of the upper suspension was transferred to another tube and the O.D was measured. Aggregation was expressed as 1- (O.D upper suspension/ O.D total bacterial suspension) $\times 100$.

\section{Adherence of mycobacterium on buccal epithelial cells}

Cell adherence assay were developed on Mycobacteria using a protocol described previously [15]. Author's own Buccal Epithelial Cells (BECs) were collected (voluntary donation) from mouth cavity by swapping with cotton bud. The cells were washed 2-3 times in PBS and the pellets were then resuspended in PBS to give approximately $\left(0.5 \mathrm{OD}_{600}\right)$ by using spectrophotometer. Bacterial cells were grown in MB7H9 broth in the presence of PA at sub-inhibitory concentration and incubated overnight at $37^{\circ} \mathrm{C}$.

The culture was adjusted to allow for absorbance at $650 \mathrm{~nm}$ of approximately 0.5 . The bacteria were then washed twice in PBS and centrifuged for $10 \mathrm{~min}$ at 10,000 rpm and resuspended in PBS. The test was performed by taking equal volumes of BECs $(0.5$ $\mathrm{OD}_{600}$ ) and bacterial suspensions that were mixed and incubated under shaking $(120 \mathrm{rpm})$ at $37^{\circ} \mathrm{C}$ for $2-3 \mathrm{hrs}$. After incubation, 2-3 $\mu \mathrm{l}$ of carbol fuchsin dye to stain mycobacterium cells and crystal violet to stain epithelial cell were added to each tube and the mixture was gently shaken. $10 \mu \mathrm{l}$ of the stained suspension were transferred to a glass slide, covered with cover-slip and examined under light microscope at $40 \mathrm{xs}$.

\section{Results and Discussion}

\section{PA Acts as effective anti-mycobacterial agent against M. smegmatis and MTB}

Natural products primarily from plants are unique resources that provide a diverse array of applications and have been used in folklore for the treatment of various diseases. In fact, over $60 \%$ and $75 \%$ of drugs currently used to treat cancer and infections respectively were derived from natural products or depended upon a natural product for their development [16]. The anti-mycobacterial effect of many natural compounds such as terpenes, alkaloids, polyketides, steroids, flavonoids, and peptides have been described [17]. Our group has already established the antifungal activity of PA against human fungal pathogen, C. albicans [11]. To analyze the anti-mycobacterial effect of PA, drug susceptibility testing was performed by two independent methods viz. broth microdilution assay determining minimum inhibitory concentration $\left(\mathrm{MIC}_{80}\right)$ and spot assays. Through broth microdilution assay, it was confirmed that PA showed anti-mycobacterial activity against $M$. smegmatis at the concentration of $350 \mathrm{\mu gml}^{-1}$ (Figure 2a). Spot assay also corresponds with the broth microdilution assay result and depicts the anti-mycobacterial effect of PA (Figure 2b). Thus, both drug susceptibility testing results indicated that PA is efficient antimycobacterial agent against M. smegmatis. We further tested the potential of PA on human pathogen MTB and to our expectation PA was able to inhibit the growth at concentration of $350 \mu \mathrm{gml}^{-1}$. The MICs of INH against M. smegmatis and MTB were found to be $4 \mathrm{ggml}^{-1}$ and $0.5 \mathrm{\mu gml}^{-1}$ respectively which were used as positive controls (Figure 2a).

\section{PA does not affect multidrug efflux transporter activity instead showed enhanced passive diffusion}

The role of multi-drug efflux pumps and particularly of the superfamily ATP Binding Cassette (ABC) are believed to be implicated in the development of natural and induced drug resistance in MTB [5]. Of the several mechanisms responsible for the development of drug resistance such as altered membrane permeability, target alteration, drug metabolism, over expression of efflux pumps which imparts resistance to broad range of antibiotics like fluoroquinolone, ofloxacin, tetracycline and INH is

(a)

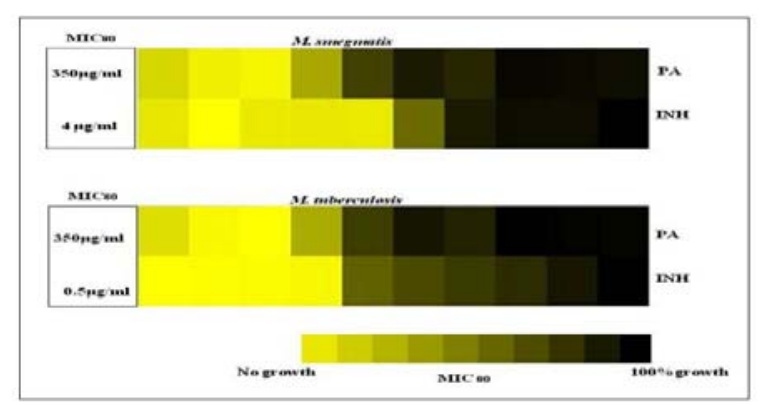

(b)



Figure 2: Drug susceptibility assays against $M$. smegmatis. (a) Broth microdilution assay to determine the $\mathrm{MIC}_{80}$ of $M$. smegmatis and MTB in presence of PA. Data was quantitatively displayed with color (see color bar), where each shade of color represents relative optical densities of the cells. INH was used as positive controls. The minimum drug concentration that inhibits growth by $80 \%$ relative to the drug-free growth control is indicated as MIC $_{80}$ (b) Spot assay of M. smegmatis in the absence (control) and presence of PA $\left(350 \mathrm{\mu gml}^{-1}\right)$. 
the major mechanism [18]. To rule out the possibility of abrogated drug efflux pump activity as possible mechanism of action for $\mathrm{PA}$, we performed the EtBr efflux assay. Our results showed that there was no significant difference $(P>0.05)$ in the EtBr efflux as estimated by extracellular EtBr concentration irrespective of the presence of PA (Figure 3a). We further accessed whether antimycobacterial activity of PA could be attributed to membrane associated changes which may involve alteration in passive diffusion of drug across the membrane. This was again achieved by estimating extracellular EtBr concentration in the presence of PA by de-energizing the cells to inactivate efflux pumps. It is evident (Figure $3 \mathrm{~b}$ ) that after $45 \mathrm{~min}$ of incubation with PA, the supernatants showed less extracellular EtBr concentration, implying enhanced passive diffusion $(P$ value $<0.05)$ of the $\mathrm{EtBr}$ in presence of PA. These results confirmed that the effect of PA on M. smegmatis is independent of the efflux pumps activity but linked with the perturbed cell membrane function.

\section{PA disrupts membrane homeostasis in M. smegmatis}

Terpenes are known to have capability to alter cell permeability and disrupt the lipid packing due to which, the membrane properties and functions may get altered. The mode of action of terpenes is closely associated with their lipophilic character preferentially influencing membrane structures which leads to increase membrane fluidity and permeability and induces disturbances in the respiration chain [18]. Enhanced passive diffusion through cell membrane in presence of PA therefore prompted us to further explore the effect of PA on cell membrane of M. smegmatis. For this we firstly performed spot assay in presence of known membrane perturbing agent such as SDS. We observed that in the presence of SDS (0.025\%), PA completely inhibited the growth of $M$. smegmatis (Figure 4a). To verify whether the anti-mycobacterial activity of PA is attributed to alteration in membrane permeability or not, we performed membrane-permeability assay using nitrocefin, a chromogenic cephalosporin substrate containing an amide bond in the $\beta$-lactam
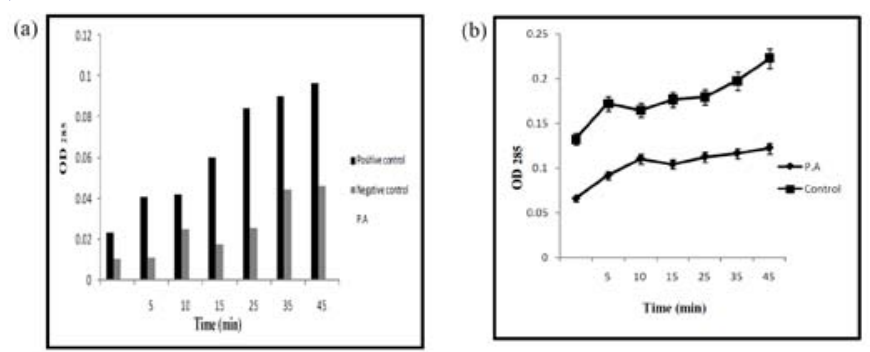

Figure 3: Effect of PA on efflux pumps and passive diffusion. (a) Extracellular concentrations of EtBr for M. smegmatis cells grown in absence (control) and presence of PA $\left(175 \mu_{\mathrm{gml}^{-1}}\right)$ calculated as described in material and methods. Negative control represents $M$. smegmatis deenergized cells without glucose. Mean of $\mathrm{OD}_{285} \pm \mathrm{SD}$ of three independent sets of experiments are depicted on $y$-axis with respect to time (minutes) on $x$ axis. (b) Passive diffusion of EtBr in absence (control) and presence of PA $\left(175 \mu \mathrm{gml}^{-1}\right)$. Mean of $\mathrm{OD}_{285} \pm$ SD of three independent sets of experiments are depicted on $Y$-axis with respect to time (minutes) on $x$-axis $(P<0.05)$. (a)

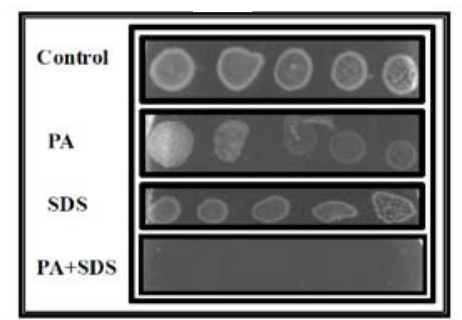

(b)

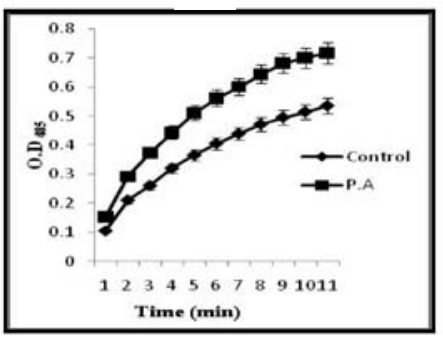

Figure 4: Effect of PA cell membrane. (a) Spot assay of M. smegmatis in the absence (control) and presence of PA $\left(175 \mu \mathrm{gmL}^{-1}\right)$ and cell membrane perturbing agent (SDS) at $0.025 \%$ (b) Nitrocefin membrane permeability assay for $M$. smegmatis cells grown in absence (control) and presence of PA $\left(175 \mu \mathrm{gml}^{-1}\right)$. Mean of $\mathrm{OD}_{485} \pm \mathrm{SD}$ of three independent sets of experiments are depicted on $Y$-axis with respect to time (minutes) on $x$-axis $(P<0.05)$.

ring, which is hydrolyzed by $\beta$-lactamase, normally localized to the bacterial periplasm [18]. Our result confirmed that there was a significant difference $(P$ value $<0.05)$ in the hydrolysis of nitrocefin as estimated by changes in absorbance in the presence of PA (Figure 4b). The disturbed membrane homeostasis of $M$. smegmatis in presence of PA clearly establishes the fact that PA has devastating effect on membrane.

\section{PA affects cell surface phenotypes of $M$. smegmatis}

M. smegmatis has been observed to exhibit important cell surface properties such as smooth colony morphology and sliding motility agar surfaces $[19,14]$. The perturbed membrane homeostasis observed from this study necessitated to study the cell surface properties. We observed that M. smegmatis cells were grown as smooth and waxy colony in contrast to PA treated cells which displayed rough and dry borders (Figure 5a). Similarly, sliding motility was impaired in presence of PA compared to the untreated cells (Figure 5b).

We further investigated the effect of PA on cell sedimentation which is inversely correlated to the hydrophobicity of the envelope $[15,20]$. Hence, we tested the effect of PA on cell sedimentation to have an estimate of any alteration in cell surface hydrophobicity. A spectrophotometric assay was used to measure the rate of cell sedimentation in $M$. smegmatis. Our observations depict that cell sedimentation rate was considerably enhanced in the cells treated with PA in comparison to the untreated cells (Figure 5c). These results confirmed the fact that PA affects cell surface phenotypes possibly due to disruption of membrane integrity. 


\section{Effect of PA on biofilm formation and cell adherence to buccal epithelial cells}

Being a natural compound and having anti-mycobacterial property, we investigated the effect of PA on M. smegmatis biofilm formation, which is a major virulence trait for pathogenicity. To study the effect of PA on biofilm formation in Mycobacterium, we performed a biofilm-formation assay using both qualitative and quantitative methods with CV. For qualitative testing, biofilm was visualized by staining control and PA treated biofilms with $\mathrm{CV}$. Compared with untreated cells, considerable reduction in biofilm formation was observed in cells treated with PA (Figure 6a). This result was validated by quantitative analysis through solubilizing the stained CV in 95\% ethanol and measuring the absorbance at $600 \mathrm{~nm}$, which reinforces the hypothesis that biofilm formation was significantly inhibited (45.33\%) in the presence of PA (Figure 6b). Since cell aggregation is linked with phenotypes such as biofilm formation $[20,21]$, we hypothesized that reduced cell aggregation in presence of PA may possibly be responsible for inhibited biofilm formation. Upon quantifying the aggregation, we found that cell aggregation was significantly lowered in PA treated cells (Figure 6c).

As a known matter of fact, cell adherence is a primary step towards mature biofilm formation. For the infection process, bacteria produce various molecules capable of promoting attachment of the bacteria to host epithelial cells. These adhesion molecules interact with host cell receptors or soluble proteins, thus acting as a link between the bacteria and the host. So, we further extended our study to verify adhesion of M. smegmatis cells on human Buccal Epithelial Cells (BECs). We observed
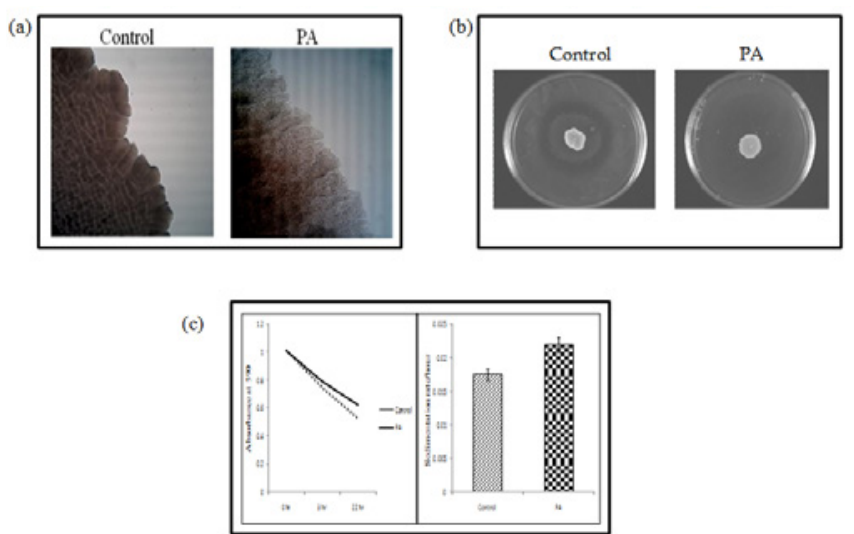

Figure 5: Effect of PA on cell surface phenotypes (a) Colony morphologies of M. smegmatis agar plates in absence (control) and presence of PA. The plates were incubated at $37^{\circ} \mathrm{C}$ for 48 hours. (b) Sliding motilities of M. smegmatis in absence (control) and presence of PA on $0.3 \%$ agarose plates. The plates were incubated at $37^{\circ} \mathrm{C}$ for 3 days. (c) Cell sedimentation of M. smegmatis. Left panel shows O.D $_{590}$ of untreated (control) and cell treated with PA $\left(175 \mu \mathrm{gmL}^{-1}\right)$ cells depicted on $y$-axis with respect to time noted at 3 and $22 \mathrm{hrs}$ on $x$-axis. Right panel shows sedimentation rates per hour on y-axis of PA treated cells with respect to control on $x$-axis, calculated by estimating the difference in $\mathrm{OD}_{590}$ from 0 till 22 hour per unit time interval. $M$ of three independent sets of experiments \pm SD (shown by error bars) are depicted. (a)
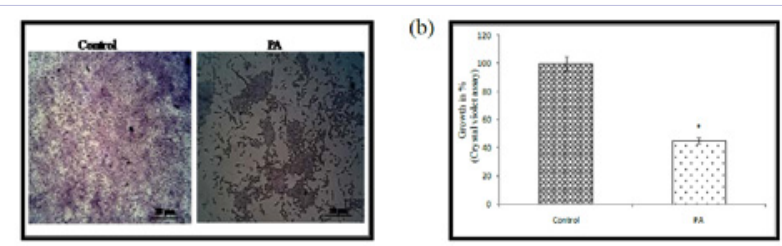

(c)
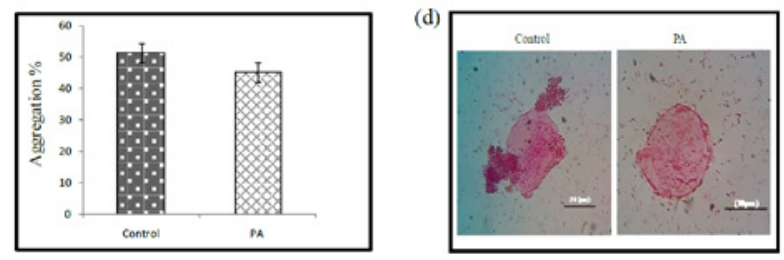

Figure 6: Effect of PA on virulence traits of $M$. smegmatis. (a) Crystal violet staining showing the biofilm formation in the absence (control) and presence of PA $\left(175 \mu \mathrm{gmL}^{-1}\right)$. (b) Effect of PA $\left(175 \mu \mathrm{gmL}^{-1}\right)$ on biofilm formation of M. smegmatis depicted as a bar graph and quantified using crystal violet dye. Mean $\mathrm{OD}_{600} \pm \mathrm{SD}$ of three independent experiments are depicted on the $\mathrm{Y}$-axis $\left({ }^{*} P<0.05\right)$. (c) Cell aggregation assay for M. smegmatis cells grown in absence (control) and presence of PA $\left(175 \mu \mathrm{gml}^{-1}\right)$. Mean of percentage aggregation \pm SD of three independent sets of experiments are depicted on $Y$-axis $(P<0.05)$. (d) Effect of PA $\left(175 \mu \mathrm{gmL}^{-1}\right)$ on cell adherence of M. smegmatis on human BECs. Left panel depicts untreated cells adhered to human $B E C S$ and right panel depicts PA treated $\left(175 \mu \mathrm{gmL}^{-1}\right)$ M. smegmatis cells not adhered to epithelial cells.

that PA treatment resulted in diminished cell adherence of $M$. smegmatis on BECs compared with functional adherence on untreated cells (Figure 6d). These results confirmed that PA is a potent inhibitor of virulence factors associated with biofilm and adhesion properties in $M$. smegmatis.

\section{PA leads to genotoxic stress in M. smegmatis}

MTB is among the most successful organism to tolerate the hostile environment within macrophages as well as environmental stresses like dehydration, low temperature and exposure to UV [22]. Although, the DNA damage response mechanisms in MTB are not well understood, it is clear that DNA repair mechanisms are vital for its persistence in the host [23]. Hence, we tested the genotoxic effect of PA on M. smegmatis. For this, we performed agar dilution assay in the presence of non lethal concentration of EtBr, a DNA damaging agent. Intriguingly, PA completely inhibited growth of the M. smegmatis in presence of EtBr suggesting possible defect in DNA repair machinery (Figure 7). Identification and characterization of new targets is the key to develop new drugs. These findings reinforce the idea of using DNA repair pathways as new drug targets. However, more studies need to be done to confirm DNA repair response machinery as effective target of PA. Taken together, this study shows that naturally occurring monoterpene, PA has promising anti-mycobacterial activity affecting multiple targets (Figure 8) in Mycobacterium hence, warrants attention for further research.

\section{Acknowledgment}

We are grateful to Prof. Sarman Singh, AIIMS, New Delhi and Prof Mandira Varma-Basil, Vallabhbhai Patel Chest Institute, 


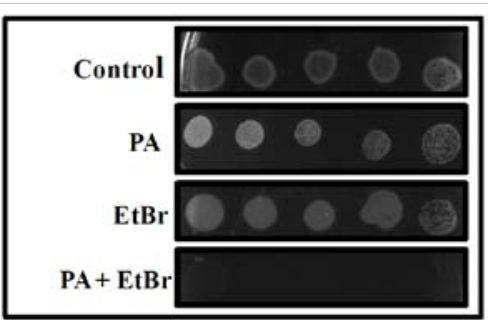

Figure 7: Genotoxic effect of PA. Spot assay of M. Smegmatis in the absence (control) and presence of PA $\left(175 \mu \mathrm{gmL}^{-1}\right)$ and DNA damaging agent (EtBr) at $13 \mu \mathrm{gml}^{-1}$.

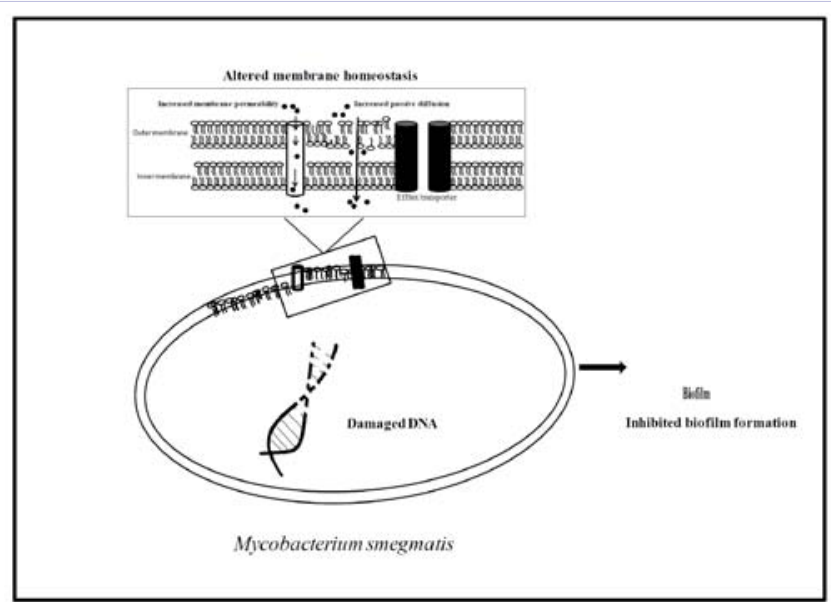

Figure 8: Summary of altered mechanisms in M. smegmatis cells after PA treatment.

University of Delhi, for providing M. smegmatis $\mathrm{mc}^{2} 155$ and $\mathrm{H}_{37}$ reference strains as generous gifts respectively.

\section{References}

1. Zumla A, George A, Sharma V, Herbert N, Baroness Masham of Ilton. WHO's 2013 global report on tuberculosis: successes, threats, and opportunities. Lancet. 2013;382(9907):1765-1767. doi: 10.1016/ S0140-6736(13)62078-4.

2. Hu Y, Wu L, Li D, Zhao Q, Jiang W, Xuet B. Association between cytokine gene polymorphisms and tuberculosis in a Chinese population in Shanghai: a case-control study. MBC Immunology. 2015;16(8):1-10. doi: 10.1186/s12865-015-0071-6.

3. Yew WW, Leung CC. Antituberculosis drugs and hepatotoxicity. Respirology. 2006;11(6):699-707.

4. Tanwar J, Das S, Fatima Z, Hameed S. Multidrug resistance: an emerging crisis. Interdiscip Perspect Infect Dis. 2014:1-7.

5. Pal R, Fatima Z, Hameed S. Efflux pumps in drug resistance of Mycobacterium tuberculosis: A panoramic view. Int J Curr Microbiol App Sci. 2014;3(8):528-546.

6. Saibabu V, Fatima Z, Khan LA, Hameed S. Therapeutic potential of dietary phenolic acids. Adv Pharmacol Sci. 2015:1-10.

7. Gupta R, Thakur B, Singh P, Singh HB, Sharma VD, Katoch VM, et al
Anti-tuberculosis activity of selected medicinal plants against multidrug resistant Mycobacterium tuberculosis isolates. Indian J Med Res. 2010;131:809-813.

8. Crowell PL. Prevention and therapy of cancer by dietary mono terpenes. J Nutr. 1999;129(3):775-778

9. Tabassum R, Vaibhav K, Shrivastava P, Khan A, Ahmed ME, Ashafaq MP, et al. Perillyl alcohol improves functional and histological outcomes against ischemia-reperfusion injury by attenuation of oxidative stress and repression of COX-2, NOS-2 and NF- $\mathrm{kB}$ in middle cerebral artery occlusion rats. Eur J Pharmacol. 2014;15:190-199. doi: 10.1016/j.ejphar.2014.09.015.

10.US Food and Drug Administration. 21CFR172.515 Code of Federal Regulations Title 21. Part 172: Food additives permitted for direct addition to food for human consumption. 2014.

11. Ansari MA, Fatima Z, Hameed S. Anticandidal Effect and Mechanisms of Monoterpenoid, Perillyl Alcohol against Candida albicans. PLoS One. 2016;11(9):e0162465. doi: 10.1371/journal.pone.0162465.

12. Clinical and Laboratory Standards Institute (CLSI). Susceptibility Testing of Mycobacteria, Nocardiae, and Other Aerobic Actinomycetes. 2003;31.

13. Pal R, Hameed S, Fatima Z. Iron deprivation affects drug susceptibilities of Mycobacteria targeting membrane integrity. J Pathog. 2015:1-10.

14. Pal R, Hameed H, Sharma S, Fatima Z. Influence of iron deprivation on virulence traits of mycobacteria. Braz J Infect Dis. 2016;20(6):585591. doi: 10.1016/j.bjid.2016.08.010.

15. Sharma S, Pal R, Hameed S, Fatima Z. Antimycobacterial mechanism of vanillin involves disruption of cell-surface integrity; virulence attributes, and iron homeostasis. 2016.

16. Tekwu ME, Askun T, Kuete V, Nkengfack AE, Nyasse B, Etoa F-X, Beng PV. Antibacterial activity of selected Cameroonian dietary spices ethnomedically used against strains of Mycobacterium tuberculosis. J Ethnopharmacol. 2012;142(2):374-382. doi: 10.1016/j. jep.2012.05.003.

17.García A, Bocanegra-Garcia V, Palma-Nicolas, Rivera G. Recent advances in antitubercular natural products. Eur J Med Chem. 2012;49:1-23. doi: 10.1016/j.ejmech.2011.12.029.

18. O'Callaghan $\mathrm{CH}$, Morris A. Inhibition of $\beta$-Lactamases by $\beta$-Lactam Antibiotics. Antimicrob Agents Chemother. 1972;2 (6):442-448.

19. Gupta KR, Kasetty S, Chatterji D. Novel functions of (p)ppGpp and cyclic di-GMP in mycobacterial physiology revealed by phenotype microarray analysis of wild-type and isogenic strains of Mycobacterium smegmatis. Appl Environ Microbiol. 2015;81(7):2571-2578.

20. Jamet S, Slama N, Domingues J, Laval F, Texier P, Eynard N et al. The nonessential mycolic acid biosynthesis genes hada and hadc contribute to the physiology and fitness of mycobacterium smegmatis. PLoS One. 2015;10(12):e0145883. doi: 10.1371/journal.pone.0145883.

21. Kragh KN, Hutchison JB, Melaugh G, Rodesney C, Roberts AEL, Irief $Y$ et al. Role of multicellular aggregates in biofilm formation. MBio. 2016;7(2);1-11.

22. Manganelli R, Provvedi R, Rodrigue S, Beaucher J, Gaudreau L, Smith I. Sigma factors and global gene regulation in Mycobacterium tuberculosis. J Bacteriol. 2004;186(4):895-902.

23. Mizrahi V, Andersen SJ. DNA repair in Mycobacterium tuberculosis. What have we learnt from the genome sequence? Mol Microbio. 1988;29(6):1331-1339. 